INP $1728 / \mathrm{PH}$

\title{
Melting of the quark condensate in the NJL model with meson loops
}

\author{
Wojciech Florkowski and Wojciech Broniowski \\ H. Niewodniczański Institute of Nuclear Physics, PL-31342 Kraków, Poland
}

\begin{abstract}
Temperature dependence of the quark condensate is studied in the Nambu-JonaLasinio model with meson loops. Substantial differences are found compared to the results with quark loops only.
\end{abstract}

\section{Introduction}

It is generally believed that QCD undergoes chiral restoration at sufficiently high temperatures. This is supported by lattice simulations [1], as well as by a variety of model calculations. As the temperature grows, the value of the quark condensate increases from its negative $T=0$ value and approaches zero. As shown in Ref. [2], in the exact chiral limit (zero current quark masses) chiral symmetry dictates the form of the first two terms $\left(\sim T^{2}\right.$ and $\left.T^{4}\right)$ in the low-temperature expansion of the quark condensate. At higher temperatures we do not have fundamental knowledge of the behavior of $\langle\bar{q} q\rangle$, however most of model calculations show a phase transition at temperatures $T \sim 150-200 \mathrm{MeV}$. Lattice calculations also show a dramatic change of $\langle\bar{q} q\rangle$ at similar temperatures.

In this letter we study the temperature dependence of the quark condensate in the two-flavor Nambu-Jona-Lasinio (NJL) model [3]. There have already been several studies [4-6] of chiral restoration in this model. Our investigation brings a new important element: it includes meson loops in a self-consistent

\footnotetext{
* Research supported in part by the Polish State Committee for Scientific Research, grant 2P03B 188 09, by the Stiftung für Deutsch-Polnische Zusammenarbeit, project 1522/94/LN, and by the Maria Skłodowska-Curie grant PAA/NSF-94-158
} 
way. Previous studies have been performed at the quark-loop level only. Attempts have been made to include meson loops in the NJL model, but selfconsistency was not completely fulfilled [7]. Our approximation is symmetryconserving $[8,9]$, hence it is consistent with all requirements of chiral symmetry. The key ingredient is the self-consistency in solving the equation for the scalar density with meson loops present. This makes the approach consistent with the requirements of chiral symmetry, such as the Goldstone theorem, Gell-Mann-Oaks-Renner and Goldberger-Treiman relations, or one-loop chiral expansions.

We find important qualitative and quantitative differences in the temperature dependence of the quark condensate in our calculation with meson loops compared to the case with quark loops only. With quark loops only, at low temperatures the condensate remains flat, whereas in our case it changes considerably. We show that in the exact chiral limit the change agrees with the prediction of the chiral perturbation theory [2]. We also find that meson loops decrease the temperature of chiral restoration by about $10 \%$.

\section{Definition of the model}

The Lagrangian of the two-flavor NJL model with scalar-isoscalar and pseudoscalar-isovector interactions is

$$
\mathcal{L}=\bar{q}\left(\mathrm{i} \partial^{\mu} \gamma_{\mu}-m\right) q+\frac{1}{2 a^{2}}\left((\bar{q} q)^{2}+\left(\bar{q} \mathrm{i} \gamma_{5} \boldsymbol{\tau} q\right)^{2}\right)
$$

where $q$ is the quark field, $m$ is the current quark mass, and $1 / a^{2}$ is the coupling constant. It is convenient to apply the formalism of effective action [10] to Lagrangian (1). Details of this procedure are given in Ref. [9]. Meson fields are introduced in the usual way (partial bosonization), with $\Phi=\left(\Phi_{0}, \boldsymbol{\Phi}\right)$ related to the sigma and the pion mean field. At the quark-loop level the effective action is

$$
I(\Phi)=\int d^{4} x\left(\frac{1}{2} a^{2} \Phi^{2}-a^{2} m \Phi_{0}+\frac{1}{2} a^{2} m^{2}\right)-\frac{1}{2} \operatorname{Tr} \ln \left(D^{\dagger} D\right)
$$

where $D$ is the Dirac operator, $D=\partial_{\tau}-\mathrm{i} \boldsymbol{\alpha} \cdot \boldsymbol{\nabla}+\beta \Phi_{0}+\mathrm{i} \beta \gamma_{5} \boldsymbol{\tau} \cdot \boldsymbol{\Phi}$. We work in Euclidean space-time $(\tau, \boldsymbol{x})$. In Eq. $(2)$ we have replaced the usual $\operatorname{Tr} \ln D$ term with $\frac{1}{2} \operatorname{Tr} \ln \left(D^{\dagger} D\right)$, which is allowed in the absence of anomalies. In fact, this replacement is necessary for the introduction of the proper-time regulator [11] used in many NJL calculations, and also in this paper. 
Meson loops bring an additional term to the effective action $[9,10]$

$$
\Gamma(\Phi)=I(\Phi)+\frac{1}{2} \operatorname{Tr} \ln \left(K^{-1}\right) .
$$

The inverse meson propagator matrix $K$ is defined as $K_{a b}^{-1}(x, y)=\frac{\delta^{2} I(\Phi)}{\delta \Phi_{a}(x) \delta \Phi_{b}(y)}$. In Eqs. $(2,3) \operatorname{Tr}$ denotes the full trace, including functional space, isospin, and in addition color and spinor trace for quarks. In the $N_{c}$-counting scheme, the quark loop term $I(\Phi)$ is the leading contribution of order $\mathcal{O}\left(N_{c}\right)$, and the meson loop term $\frac{1}{2} \operatorname{Tr} \ln K$ is of order $\mathcal{O}(1)$. Thus the one-meson-loop contributions give the first correction to the leading- $N_{c}$ results.

Using standard methods, Green's functions can be obtained from Eq. (3) via differentiation with respect to mean meson fields. Of particular importance is the one-point function, which gives the expectation value of the sigma field. The condition

$$
\begin{aligned}
{\frac{\delta \Gamma(\Phi)}{\delta \Phi_{0}(x)}}_{\mid \Phi_{0}(x)=S}= & a^{2}(S-m)-\frac{1}{2} \operatorname{Tr}\left(\left(D^{\dagger} D\right)^{-1} \frac{\delta\left(D^{\dagger} D\right)}{\delta \Phi_{0}(x)}\right)_{\Phi_{0}(x)=S} \\
& +\frac{1}{2} \operatorname{Tr}\left(K \frac{\delta K^{-1}}{\delta \Phi_{0}(x)}\right)_{\Phi_{0}(x)=S}=0
\end{aligned}
$$

yields the equation for the vacuum expectation value of $\Phi_{0}$, which we denote by $S$. Introducing

$$
\begin{aligned}
& K_{\sigma}\left(S, Q^{2}\right)=\left(4 N_{c} f\left(S, Q^{2}\right)\left(Q^{2}+4 S^{2}\right)+a^{2} m / S\right)^{-1} \\
& K_{\pi}\left(S, Q^{2}\right)=\left(4 N_{c} f\left(S, Q^{2}\right) Q^{2}+a^{2} m / S\right)^{-1}
\end{aligned}
$$

and retaining terms up to order $\mathcal{O}\left(N_{c}^{0}\right)$, Eq. (4) can be written in the form [9]

$$
\begin{aligned}
& a^{2}(S-m)-8 N_{c} S g(S) \\
& +S \frac{N_{c}}{4 \pi^{4}} \int d^{4} Q\left\{\left[2 f(S, 0)+\frac{d}{d S^{2}}\left(f\left(S, Q^{2}\right)\left(Q^{2}+4 S^{2}\right)\right)\right] K_{\sigma}\left(S, Q^{2}\right)\right. \\
& \left.+3\left[2 f(S, 0)+\frac{d}{d S^{2}} f\left(S, Q^{2}\right) Q^{2}\right] K_{\pi}\left(S, Q^{2}\right)\right\}=0 .
\end{aligned}
$$

Functions $g$ and $f$ in the above expressions are the quark bubble functions. Their form is very simple if no cut-offs were present. In this case we would have $g(S)=\int \frac{d^{4} k}{(2 \pi)^{4}} \frac{1}{k^{2}+S^{2}}$ and $f\left(S, Q^{2}\right)=\int \frac{d^{4} k}{(2 \pi)^{4}} \frac{1}{k^{2}+S^{2}} \frac{1}{(k+Q)^{2}+S^{2}}$, and Eq. (6) could be interpreted via standard Feynman diagrams (see Fig. 1). In the presence of a 
cut-off these functions are complicated. In the case of the proper-time cut-off [11] used here we have [9]

$$
g(S)=\int \frac{d^{4} k}{(2 \pi)^{4}} \int_{\Lambda_{f}^{-2}}^{\infty} d s \exp \left\{-s\left[k^{2}+S^{2}\right]\right\}=\frac{\Lambda_{f}^{2}}{16 \pi^{2}} E_{2}\left[\frac{S^{2}}{\Lambda_{f}^{2}}\right]
$$

and

$$
\begin{aligned}
f\left(S, Q^{2}\right) & =\int \frac{d^{4} k}{(2 \pi)^{4}} \int_{\Lambda_{f}^{-2}}^{\infty} d s s \int_{0}^{1} d u \exp \left\{-s\left[k^{2}+S^{2}+u(1-u) Q^{2}\right]\right\} \\
& =\frac{1}{16 \pi^{2}} \int_{0}^{1} d u E_{1}\left[\frac{S^{2}}{\Lambda_{f}^{2}}+u(1-u) \frac{Q^{2}}{\Lambda_{f}^{2}}\right],
\end{aligned}
$$

where $\Lambda_{f}$ is the quark cut-off, and the exponential integral is defined as $E_{n}(x) \equiv \int_{1}^{\infty} d t \frac{e^{-x t}}{t^{n}}$

The one meson-loop gap equation (6) requires also the introduction of a regulator for meson momenta. In other words, we have to regularize the divergent integral over $d^{4} Q$. In Ref. [9] this was achieved by the substitution $\int d^{4} Q \longrightarrow \pi^{2} \int_{0}^{\Lambda_{b}^{2}} d Q^{2} Q^{2}$, where $\Lambda_{b}$ was the four-dimensional Euclidean meson momentum cutoff. In the present study at finite temperatures, we employ the three-dimensional cutoff procedure, i.e., we make the replacement

$$
\int d^{4} Q \longrightarrow 4 \pi \int d \omega \int_{0}^{\Lambda_{b}} d q q^{2}
$$

where $Q=(\omega, \mathbf{q})$ and $q=|\mathbf{q}|$. The form (9) is convenient for the implementation of the boundary conditions satisfied by temperature Green's functions. 


\section{Finite temperature}

For calculations at finite temperature $T$ we shall adopt the imaginary time formalism [12]. This can be done by making the following replacement in the quark momentum integrals

$$
\int \frac{d^{4} k}{(2 \pi)^{4}} F(k)=\int \frac{d E}{2 \pi} \int \frac{d^{3} k}{(2 \pi)^{3}} F(E, \mathbf{k}) \rightarrow T \sum_{j=-\infty}^{\infty} \int \frac{d^{3} k}{(2 \pi)^{3}} F\left(E_{j}, \mathbf{k}\right) .
$$

Here $F(k)=F(E, \mathbf{k})$ is an arbitrary integrand, and the sum runs over the fermionic Matsubara frequencies $E_{j}=(2 j+1) \pi T$. The integral over the meson four-momenta should be also replaced by the sum of the form (10). In this case, however, the sum runs over the bosonic Matsubara frequencies $\omega_{n}=2 \pi n T$. With this prescription we can turn to the calculation of the functions which are the finite temperature analogs of $g(S)$ and $f\left(S, Q^{2}\right)$. We find

$$
\begin{aligned}
g(S, T) & =T \sum_{j} \int \frac{d^{3} k}{(2 \pi)^{3}} \int_{\Lambda_{f}^{-2}}^{\infty} d s \exp \left\{-s\left[E_{j}^{2}+\mathbf{k}^{2}+S^{2}\right]\right\} \\
& =\frac{T \Lambda_{f}}{8 \pi^{\frac{3}{2}}} \sum_{j} E_{\frac{3}{2}}\left[\frac{S^{2}+E_{j}^{2}}{\Lambda_{f}^{2}}\right]
\end{aligned}
$$

and

$$
\begin{aligned}
& f(S, n, q, T)=T \sum_{j} \int \frac{d^{3} k}{(2 \pi)^{3}} \int_{\Lambda_{f}^{-2}}^{\infty} d s s \int_{0}^{1} d u \times \\
& \exp \left\{-s\left[S^{2}+u(1-u)\left(\omega_{n}^{2}+\mathbf{q}^{2}\right)+[\mathbf{k}-\mathbf{q}(1-u)]^{2}+\left[E_{j}-\omega_{n}(1-u)\right]^{2}\right]\right\}
\end{aligned}
$$
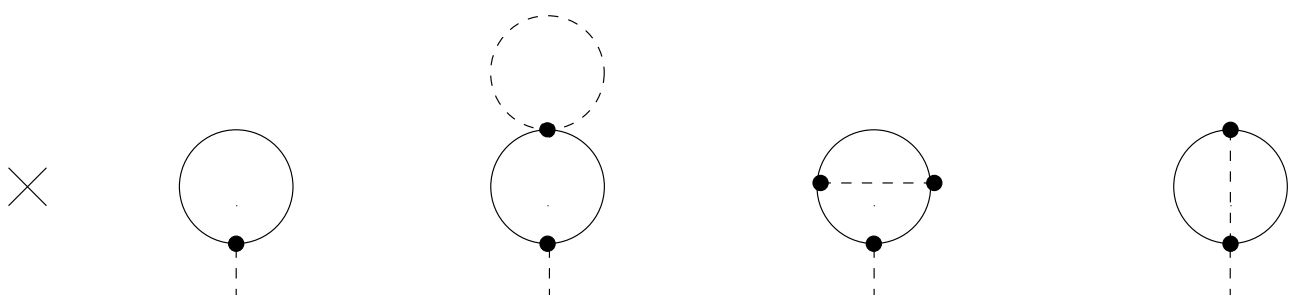

Fig. 1. Diagramatic representation of Eq. (6). The cross represents the first term, the one-quark-loop contribution corresponds to the second term, and meson-loop terms represent subsequent terms. The solid lines represent the quark propagator $1 /\left(D^{\dagger} D\right)$, the dased lines correspond to the meson propagators $K$ of Eqs. (5), the external dased line represents scalar-isoscalar coupling, and the vertices follow from the form of $\left(D^{\dagger} D\right)$. 


$$
=\frac{T}{8 \pi^{\frac{3}{2}} \Lambda_{f}} \sum_{j} \int_{0}^{1} d u E_{\frac{1}{2}}\left[\frac{S^{2}}{\Lambda_{f}^{2}}+u(1-u) \frac{\omega_{n}^{2}+\mathbf{q}^{2}}{\Lambda_{f}^{2}}+\frac{\left[E_{j}-\omega_{n}(1-u)\right]^{2}}{\Lambda_{f}^{2}}\right] .
$$

Analogously, the inverse meson propagators become

$$
\begin{aligned}
& K_{\sigma}(S, n, q, T)=\left(4 N_{c} f(S, n, q, T)\left(\omega_{n}^{2}+\mathbf{q}^{2}+4 S^{2}\right)+a^{2} m / S\right)^{-1} \\
& K_{\pi}(S, n, q, T)=\left(4 N_{c} f(S, n, q, T)\left(\omega_{n}^{2}+\mathbf{q}^{2}\right)+a^{2} m / S\right)^{-1} .
\end{aligned}
$$

Finally, we can write the finite-temperature analog of Eq. (6):

$$
\begin{aligned}
& a^{2}(S-m)-8 N_{c} S g(S, T)+\frac{2 S N_{c} T}{\pi^{2}} \sum_{n} \int_{0}^{\Lambda_{b}} d q q^{2} \times \\
& \left\{\left[2 f(S, 0,0, T)+\frac{d}{d S^{2}}\left(f(S, n, q, T)\left(\omega_{n}^{2}+\mathbf{q}^{2}+4 S^{2}\right)\right)\right] K_{\sigma}(S, n, q, T)\right. \\
& \left.+3\left[2 f(S, 0,0, T)+\frac{d}{d S^{2}} f(S, n, q, T)\left(\omega_{n}^{2}+\mathbf{q}^{2}\right)\right] K_{\pi}(S, n, q, T)\right\}=0 .
\end{aligned}
$$

If chiral symmetry is broken, then the above equation has a nontrivial solution for $S$. The quark condensate and $S$ are related by the formula

$$
\langle\bar{q} q\rangle=-a^{2}(S-m),
$$

which follows immediately from the fact that $\langle\bar{q} q\rangle=\delta \Gamma(\Phi) / \delta m$ and Eq. (3).

\section{Low-temperature expansion in the chiral limit}

Before presenting our numerical results for $\langle\bar{q} q\rangle_{T}$ let us consider the lowtemperature expansion. As shown by Gasser and Leutwyler [2], in the chiral limit the low-temperature expansion of the quark condensate has the form

$$
\langle\bar{q} q\rangle_{T}=\langle\bar{q} q\rangle_{0}\left(1-\frac{T^{2}}{8 F_{\pi}^{2}}-\frac{T^{4}}{384 F_{\pi}^{4}}+\ldots\right) .
$$

First, let us do the $N_{c}$ counting in this formula. Since $F_{\pi} \sim \mathcal{O}\left(\sqrt{N_{c}}\right)$, subsequent terms in the expansion are suppressed by $1 / N_{c}$. Since our one-mesonloop calculation accounts for first subleading effects in the $1 / N_{c}$ expansion, we 
can hope for reproducing only the $T^{2}$ term in Eq. (16). Further terms would require more loops.

Using standard techniques [12], the sum over the bosonic Matsubara frequencies in Eq. (14) can be converted to a contour integral in the complex energy plane. By deforming this contour we collect all contributions from the singularities of the integrand, weighted with the thermal Bose distribution. At low temperatures, the dominant contribution comes from the lowest lying pion pole, and other singularities are negligible. Thus, the third term in (14) becomes

$$
\frac{3 T}{\pi^{2}} \sum_{n} \int_{0}^{\Lambda_{b}} d q q^{2} \frac{1}{\omega_{n}^{2}+q^{2}}=\frac{3}{2 \pi^{2}} \int_{0}^{\Lambda_{b}} d q q\left[1+\frac{2}{e^{q / T}-1}\right] .
$$

Writing Eq. (17) we have approximated the function $f(M, n, q, T)$, appearing in the pion propagator, by its value at $n=q=0$. For sufficiently large cutoff $\Lambda_{b}$, the integral over the thermal distribution function in (17) can be expressed by the Riemann zeta function $\zeta(2)=\pi^{2} / 6$. Thus, the final result for (17) is $3 \Lambda_{b}^{2} / 4 \pi^{2}+T^{2} / 2$. Inserting the above result into the gap equation (14) we find, with $m=0$, the following equality:

$$
h(S, T) \equiv a^{2}-8 N_{c} g(S, T)+\frac{3 \Lambda_{b}^{2}}{4 \pi^{2}}+\frac{1}{2} T^{2}=0 .
$$

Eq. (18) defines implicitly the function $S(T)$, which satisfies the equation

$$
\frac{d S}{d T^{2}}=-\frac{\partial h(S, T) / \partial T^{2}}{\partial h(S, T) / \partial S}=\left[16 N_{c} \frac{\partial g(S, T)}{\partial S}\right]^{-1} .
$$

Here we have neglected the term $\partial g(S, T) / \partial T^{2}$, since it is exponentially suppressed by the factor $\exp (-S / T)$. Furthermore, the leading $-N_{c}$ term on the right hand side of (19) can be rewritten using the relations [9] $\partial g(S, T) / \partial S=$ $-2 S f(S, 0)$ and $4 N_{c} f(S, 0)=\bar{F}_{\pi}^{2} / S^{2}$, where $\bar{F}_{\pi}$ is the leading- $N_{c}$ piece of the pion decay constant. Collecting these equalities we arrive at $d S / d T^{2}=$ $-S /\left(8 \bar{F}_{\pi}^{2}\right)$, which finally gives

$$
S(T)=S(0)\left[1-\frac{T^{2}}{8 \bar{F}_{\pi}^{2}}\right] .
$$

Proportionality (15) implies that the above expression coincides (in the large $N_{c}$ limit) with Eq. (16). Hence our method is consistent with a basic requirement of chiral symmetry at the one-meson-loop level. 


\section{Results}

In the exact chiral limit the model has 3 parameters: $a, \Lambda_{f}$, and $\Lambda_{b}$. In this paper we fix arbitrarily $\Lambda_{b} / \Lambda_{f}=\frac{1}{2}$. The remaining 2 parameters are fixed by reproducing the physical value of $F_{\pi}=93 \mathrm{MeV}$ and a chosen value for $\langle\bar{q} q\rangle_{0}$. For the case of $m \neq 0$ we have an extra parameter, $m$, which is fitted by requiring that the pion has its physical mass. We compare results with meson loops to results with the quark loop only $\left(\Lambda_{b}=0\right)$. Parameters for the two calculations are adjusted in such a way, that the values of $F_{\pi},\langle\bar{q} q\rangle_{0}$, and $m_{\pi}$ are the same.

The calculation of $F_{\pi}$ with meson loops, although straightforward, is rather tedious, so we do not present it here. The method has been presented in detail in Ref. $[9,13]$. The only difference in our calculation is that the threemomentum cut-off (9) rather than the four-momentum cut-off of Ref. [9] is used.

Figure 2 shows the dependence of $\langle\bar{q} q\rangle$ on $T^{2}$. The solid line represents the case with meson loops. We note that at low temperatures the curve has a finite slope, as requested by Eq. (20). The slope is close to the leading-order Gasser-Leutwyler result (dotted curve). As explained earlier, the slopes would overlap in the large- $N_{c}$ limit. This behavior is radically different from the case with quark loops only (dashed curve). In this case at low temperatures $\langle\bar{q} q\rangle_{T}-\langle\bar{q} q\rangle_{0} \sim e^{-M / T}$, where $M$ is the mass of the constituent quark. All derivatives of this function vanish at $T=0$, and $\langle\bar{q} q\rangle$ is flat at the origin. We can also see from the figure that the fall-off of the condensate is faster when the meson loops are included. In fact, for the parameters of Fig. 2 we have an interesting phenomenon. At $T=162 \mathrm{MeV}$ the condensate abruptly jumps to 0 . There is a first-order phase transition, with a latent heat necessary to melt the quark condensate. Such a behavior is not present in the case of calculations without meson loops [4-6]. We note that with meson loops present the chiral restoration temperature is $162 \mathrm{MeV}$, i.e. about $10 \%$ less than $176 \mathrm{MeV}$ of the quark-loop-only case.

Figure 3 shows the same study, but for the physical value of $m_{\pi}$. We note that now $\langle\bar{q} q\rangle$ (solid line) is also flat at the origin, since the pion is no more massless, and at low $T$ we have $\langle\bar{q} q\rangle_{T}-\langle\bar{q} q\rangle_{0} \sim e^{-m_{\pi} / T}$. Nevertheless, the region of this flatness is small, and at intermediate temperatures the curve remains close to the Gasser-Leutwyler expansion. We note again that meson loops considerably speed up the melting of the condensate compared to the case of quark loops only. However, there is no first-order phase transition such as in Fig. 2. Instead, we observe a smooth cross-over typical for the case of $m \neq 0$.

The faster change of the quark condensate in our study is not surprising. It 
is caused by the presence of light pions which are known to play a dominant role at low-temperatures [7]. The behavior of $\langle\bar{q} q\rangle$ reflects this general feature. Concluding, we stress that the inclusion of meson loops in the NJL model qualitatively and quantitatively changes the results in comparison to the calculations at the quark-loop level. In particular, we find finite slope of $\langle\bar{q} q\rangle$ vs. $T^{2}$ at the origin in the chiral limit, faster melting of the condensate, and lower chiral restoration temperature.

\section{References}

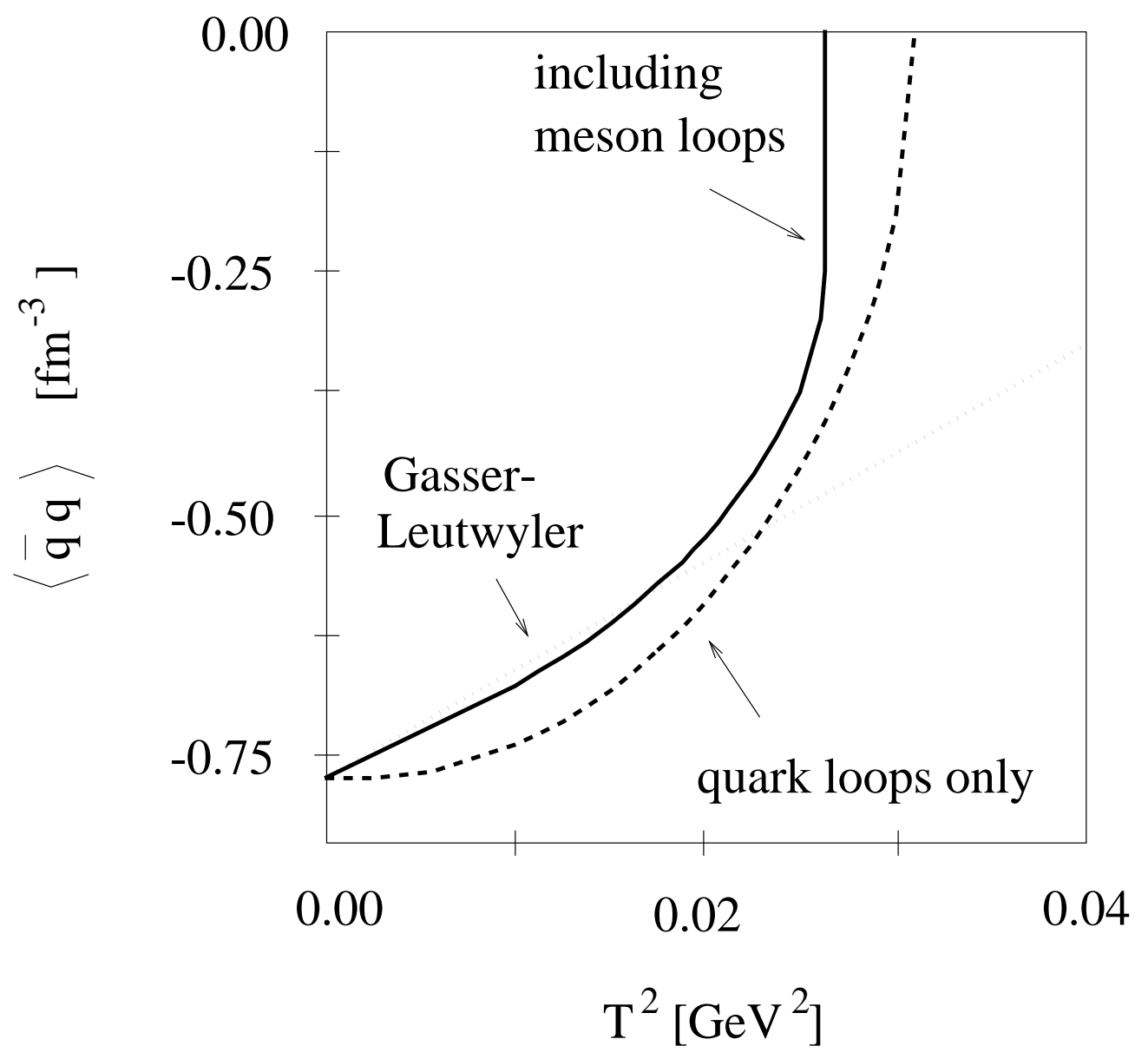

Fig. 2. Dependence of of the quark condensate on $T^{2}$ in the chiral limit $m_{\pi}=0$. The curves correspond to the calculation with meson loops (solid line), with quark loops only (dashed line), and the lowest-order chiral expansion (dotted line). The parameters for the solid line and dashed line are adjusted in such a way that $F_{\pi}=93 \mathrm{MeV}$ and $\langle\bar{q} q\rangle_{0}=-(184 \mathrm{MeV})^{3}$. For the solid line $a=175 \mathrm{MeV}, \Lambda_{f}=723 \mathrm{MeV}$, and $\Lambda_{b}=\frac{1}{2} \Lambda_{f}$, whereas for the dashed line $a=201 \mathrm{MeV}, \Lambda_{f}=682 \mathrm{MeV}$, and $\Lambda_{b}=0$. 
[1] K. Kanaya, Finite Temperature $Q C D$ on the Lattice, talk given at International Symposium on Lattice Field Theory, Melbourne, Australia, 11-15 July 1995, eprint hep-lat/9510040

[2] J. Gasser and H. Leutwyler, Phys. Lett. 184B (1987) 83; Phys. Lett. 188B (1987) 477; Nucl. Phys. B307 (1988) 763

[3] Y. Nambu and G. Jona-Lasinio, Phys. Rev. 122 (1961) 345

[4] T. Hatsuda and T. Kunihiro, Phys. Rev. Lett. 55 (1985) 158

[5] V. Bernard, U.-G. Meissner, and I. Zahed, Phys. Rev. D36 (1987) 819

[6] M. Lutz, S. Klimt, and W. Weise, Nucl. Phys. A542 (1992) 521

[7] P. Zhuang, J. Hüfner, and S. P. Klevansky, Nucl. Phys. A576 (1994) 525; P. Zhuang, J. Hüfner, S. P. Klevansky, and H. Voss, Ann. Phys. (N.Y.) 234 (1994) 225

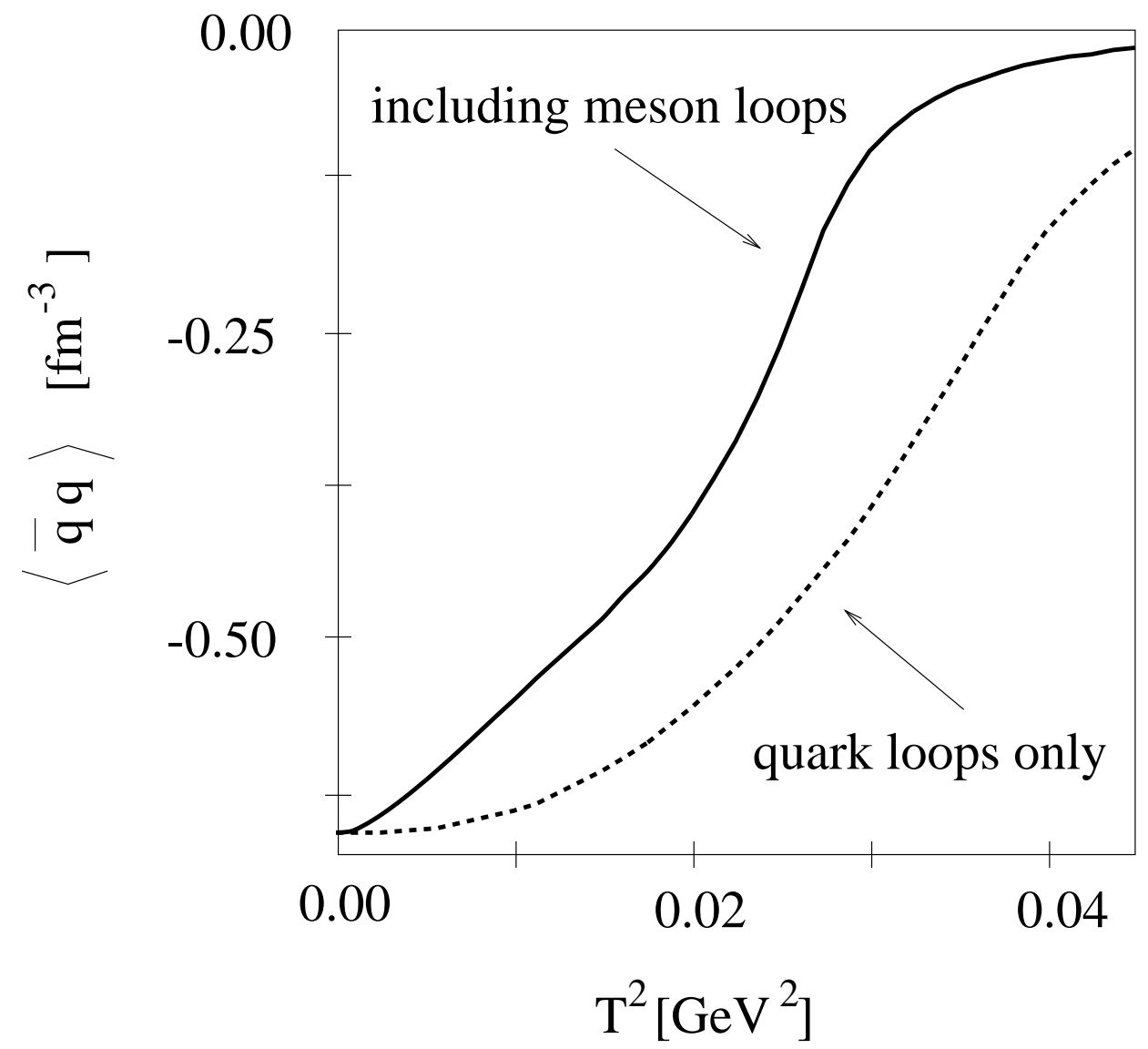

Fig. 3. Same as Fig. 2 for $m_{\pi}=139 \mathrm{MeV}, F_{\pi}=93 \mathrm{MeV}$, and $\langle\bar{q} q\rangle_{0}=-(174 \mathrm{MeV})^{3}$. For the solid line $a=164 \mathrm{MeV}, \Lambda_{f}=678 \mathrm{MeV}, \Lambda_{b}=\frac{1}{2} \Lambda_{f}$, and $m=15 \mathrm{MeV}$, whereas for the dashed line $a=175 \mathrm{MeV}, \Lambda_{f}=645 \mathrm{MeV}, \Lambda_{b}=0$, and $m=15 \mathrm{MeV}$. 
[8] V. Dmitrašinović, H.-J. Schulze, R. Tegen, and R. H. Lemmer, Ann. of Phys. (NY) 238 (1995) 332

[9] E.N. Nikolov, W. Broniowski, C.V. Christov, G. Ripka and K. Goeke, Univ. of Bochum preprint No. RUB-TPH-13/95 (1995), eprint hep-ph/9602274

[10] C. Itzykson and J.-B. Zuber, Quantum Field Theory (McGraw-Hill, New York, 1980)

[11] D. I. Dyakonov and V. Y. Petrov, JETP Lett. 43 (1986) 57

[12] J. Kapusta., Finite-Temperature Field Theory (Cambridge University Press, Cambridge, 1989)

[13] E. Nikolov, Ph.D. thesis, Ruhr-Univ. Bochum, 1995 